\title{
Creating a Simulated Dataset for Training Deep Convolutional Neural Networks for Use in Cardiovascular Photoacoustic Tomography
}

\author{
Hayley Chan ${ }^{1}$, Katherine Leyba ${ }^{2}$, Craig J. Goergen, $\mathrm{PhD}^{2}$ \\ ${ }^{1}$ Indiana University School of Medicine, ${ }^{2}$ Purdue University Weldon School of Biomedical \\ Engineering
}

Background/Objective: Photoacoustic tomography possesses increasing potential as a noninvasive imaging method that combines optical and acoustic imaging to maximize the visualization of tissue. Determining the composition, orientation, and location of anatomical structures in multidimensional space requires maximizing image resolution and differentiation from noise and reflection artifacts. Using simulations to develop and improve methods for image resolution allows for flexibility and variation of numerous variables.

Methods: Binary masks were created from mouse common carotid ultrasound images using a graphical user interface for MATLAB. With the k-Wave toolbox, we performed time-reversal photoacoustic simulations using the masks. Medium properties for the simulations were assigned for sound speed and density for connective tissue $\left(1540 \mathrm{~m} / \mathrm{s}, 1027 \mathrm{~kg} / \mathrm{m}^{3}\right)$ and arterial walls $\left(1569 \mathrm{~m} / \mathrm{s}, 1102 \mathrm{~kg} / \mathrm{m}^{3}\right)$. The dataset was augmented through rotational and mirrored transformations and the addition of noise and reflection artifacts via Python open-source software.

Results: A set of 87 binary masks was generated from common carotid ultrasound images. These masks were used to simulate initial pressure distributions through the k-Wave toolbox to reconstruct the structure of the common carotid. Each simulation yielded graphs for initial pressure and sensor distribution, simulated sensor data, reconstructed initial pressure, and a comparison profile between the original and reconstructed pressure. Data augmentation was implemented using the reconstructed pressure output from the 87 simulations, each producing 12 distinct images from rotations and mirroring with the addition of noise and reflection artifacts. The final dataset yielded 1044 images.

Conclusion and Potential Impact: Future work will involve applying this dataset to a neural network to improve photoacoustic quality such that transfer learning can be applied on ex vivo and in vivo datasets. Thus, there is potential for use in diagnostic applications in patients with cardiovascular disease states like atherosclerosis and aneurysms that require high resolution visualization of tissue structure and composition. 